1 Universidade Estadual Vale do Acaraú (UVA) Sobral (CE), Brasil. elianyy@hotmail.com

2 Universidade do Porto Porto, Portugal.

fneto@fpce.up.pt

3 Universidade Estadual Vale do Acaraú (UVA) Sobral (CE), Brasil.

tamiresafelix@gmail.com

4 Universidade Estadual Vale do Acaraú (UVA) Sobral (CE), Brasil. gleisson_nega@hotmail.com

5 Universidade Estadual do Ceará (Uece) - Fortaleza (CE), Brasil.

pc49almeida@gmail.com

\section{Qualidade de vida de imigrantes brasileiras vivendo em Portugal}

\author{
Quality of life of Brazilian immigrant women living in Portugal \\ Eliany Nazaré Oliveira', Félix Fernando Monteiro Neto ${ }^{2}$, Tamires Alexandre Félix ${ }^{\mathbf{3}}$, Gleisson \\ Ferreira Lima ${ }^{4}$, Paulo César de Almeida ${ }^{5}$
}

RESUMO O artigo teve como objetivo avaliar a Qualidade de Vida (QV) de brasileiras vivendo em Portugal. O 'Medical Outcomes Study: 36-Item Short Form Survey' (SF-36) e um questionário sociodemográfico foram aplicados à amostra de 682 brasileiras maiores de 18 anos. Verificou-se que a média dos preditores de QV foi superior para a dimensão 'capacidade funcional'; enquanto as dimensões 'aspectos emocionais', 'saúde mental' e 'vitalidade' apresentaram valores menores. Constatou-se que os processos da migração e da QV constituem pauta de investigação contínua para a promoção da saúde no país de acolhimento.

PALAVRAS-CHAVE Qualidade de vida. Imigrantes. Mulheres.

ABSTRACT This article aims to evaluates the Quality of Life (QoL) of Brazilian women living in Portugal. The 'Medical Outcomes Study: 36-Item Short Form Survey' (SF-36) and a sociodemographic questionnaire were applied to the sample of 682 Brazilian women over 18 years old. The average values for the predictors of $Q$ oL were higher for the dimension 'functional capacity'; whereas 'emotional aspects', 'mental health', and 'vitality' showed lower values. It was found that the processes of migration and QoL constitute an agenda of continuous research for health promotion in the hosting country.

KEYWORDS Quality of life. Immigrants. Women. 


\section{Introdução}

Os fluxos migratórios fazem parte da história da humanidade, pois as conquistas, as dominações de povos e as colonizações de novas terras estimulam a movimentação das pessoas. A migração pode ser compreendida como um fenômeno global e multidimensional que requer profundas mudanças. Para Bäckström e Pereira (2012), compreender a questão migratória implica objetivar o que se entende por multicultural e intercultural, considerando a interculturalidade como um desafio capaz de reconhecer e valorizar a diferença. Não basta que as diferentes culturas alcancem uma convivência baseada no respeito mútuo e na solidariedade, mostra-se necessária uma interação significativa entre elas.

A literatura sobre processos migratórios tem indicado que uma parte significativa de indivíduos migra em busca de uma vida melhor, com vistas a uma Qualidade de Vida (QV) muitas vezes inalcançável em seus países de origem (CORREA ET AL., 2015; FRANKEN; COUTINHO; RAMOS, 2012; MARINUCCI, 2016; MEZZADRA, 2015). Indo ao encontro de pesquisas desenvolvidas na França, em Portugal, na Itália e em outros países, tanto europeus como norte-americanos, destaca-se o complexo e variado conjunto de fatores socioeconômicos, psicossociais e familiares observado nos contextos migratórios. Tais fatores afetam efetivamente a saúde, os níveis de estresse, o desenvolvimento e a $\mathrm{QV}$ das pessoas e das famílias migrantes, principalmente daquelas oriundas de países em desenvolvimento (RAMOS, 1993, 2004).

O conceito de 'qualidade de vida' é recente e tem sido utilizado muito frequentemente, tanto pela população como pela comunidade científica. Trata-se de tema central nas análises e nas políticas de planejamento e gestão no setor da saúde. Diversas correntes propõem uma definição para esse conceito. Para Minayo, Hartz e Buss (2000), a QV implica três fóruns de referência:
1. Histórico, ou seja, em determinado momento o desenvolvimento econômico, social e tecnológico de uma sociedade apresenta um parâmetro de QV diferente da mesma sociedade em outro momento;

2. Cultural, isto é, os valores e as necessidades são construídos e hierarquizados de modo diferente pelos povos, revelando suas tradições;

\section{Estratificação, ou classes sociais.}

Minayo, Hartz e Buss (2000) trazem o debate sobre a QV em relação a essas características mais amplas, polissêmicas, que envolvem o desenvolvimento, a democracia e o modo e estilo de vida, associando a avaliação socioeconômica à saúde das populações.

Pondera-se que a concepção de QV remete a aspectos históricos e culturais e às classes sociais, uma vez que sociedades em diferentes estágios de desenvolvimento econômico, social e tecnológico apresentam valores e necessidades distintas em termos culturais e as diversas estratificações sociais também têm percepções diferentes de QV (MINAYO; HARTZ; BUSS, 2000).

A Organização Mundial da Saúde (OMS) (WHO, 2002) define 'qualidade de vida' como um fenômeno holístico que reúne os recursos sociais, individuais e físicos necessários ao indivíduo para a concretização de seus objetivos e aspirações, bem como para a satisfação de suas demandas em diferentes níveis.

De acordo com Bäckström e Pereira (2012), mesmo havendo inúmeras definições de QV, nenhuma delas é amplamente aceita. O que fica cada vez mais evidente é que não se pode contemplar apenas fatores relacionados com a saúde, como bem-estar físico, funcional, emocional e mental; outros elementos importantes na vida das pessoas, como trabalho, família, amigos e as circunstâncias do cotidiano, também são relevantes; e a percepção pessoal sempre é primordial.

Naime (2006) alerta que o tema QV e imigração deve ser estudado e discutido em 
todas as sociedades atuais, pois os grandes fluxos populacionais se alteram com o surgimento de um grupo novo, geralmente ativo e autônomo, formado pelos imigrantes. Perotti (1997) ressalta que são múltiplos os obstáculos que as minorias étnicas enfrentam para se integrar em uma sociedade e em um universo sociocultural diferente do seu. São dificuldades de natureza material, legal e cultural que o imigrante vivencia na constante busca de uma QV melhor. Isso intensifica a movimentação de pessoas e gera novas formas de mobilidade, novos direcionamentos e novas trajetórias de migração.

Entre os novos movimentos migratórios, encontram-se aqueles que envolvem pessoas com condições necessárias à sua reprodução econômica. Em seus locais de origem, elas já não desempenham o papel de produtores diretos das mercadorias e, mesmo assim, migram em busca de uma vida melhor. A imigração brasileira em Portugal enquadra-se nesse cenário, cada vez mais presente e disseminado, com amplitude em todas as regiões do país e impacto no tecido sociocultural dos locais de acolhimento.

A partir dessa contextualização, a questão de pesquisa foi: Como se apresenta a QV de imigrantes brasileiras que vivem em Portugal, tendo como parâmetro os itens do 'Medical Outcomes Study: 36-Item Short Form Survey' (SF-36)? Dessa forma, o objetivo deste artigo foi avaliar a QV dessas imigrantes brasileiras com base nesse referencial.

\section{Material e métodos}

Foram selecionadas para a amostra deste estudo 682 brasileiras maiores de 18 anos que viviam em Portugal há pelo menos 3 meses.

Este estudo adotou como referencial o SF-36, um instrumento genérico de avaliação da QV de fácil administração e compreensão. Ele é formado por uma questão de avaliação comparativa entre as condições de saúde atual e as de um ano atrás e 35 itens, englobados em oito escalas (ou componentes): a) capacidade funcional (10 itens); b) aspectos físicos (4 itens); c) dor (2 itens); d) estado geral de saúde (5 itens); e) vitalidade (4 itens); f) aspectos sociais (2 itens); g) aspectos emocionais (3 itens), e h) saúde mental (5 itens). Avalia tanto os aspectos negativos (doença ou enfermidade) como os positivos da saúde (bem-estar ou QV) (CICONELLI, 1997).

$\mathrm{Na}$ avaliação dos resultados, após sua aplicação, atribui-se um escore para cada questão, que posteriormente é convertido em uma escala de 0 a 100 , onde 0 corresponde ao pior estado de saúde e 100 ao melhor, com análise isolada de cada dimensão. Propositalmente, não há um único valor que resuma toda a avaliação, que se traduz em um estado geral de saúde melhor ou pior, de modo que, em uma média de valores, evite-se o erro de não identificar os verdadeiros problemas relacionados com a QV e à saúde do entrevistado ou mesmo de subestimá-los (CICONELLI, 1997).

Para a caracterização da amostra, foi adotado um questionário sociodemográfico com os seguintes itens: a) idade; b) estado civil; c) escolaridade; d) filho(s); e) situação no mercado de trabalho; f) tempo de moradia em Portugal; e g) situação de moradia.

Os escores para os domínios do SF-36 obtidos em populações adultas apresentam confiabilidade e validade satisfatórias quando comparados a outros instrumentos de avaliação da QV. Constatou-se que o SF-36 atende aos padrões psicométricos exigidos em relação à qualidade dos dados, pressupostos de escalonamento, confiabilidade e validade como instrumento; suas escalas reproduzem as dimensões físicas e mentais hipotéticas; e os padrões de relações entre os fatores e as escalas são preditivos de suas associações a critérios externos de saúde física e mental. Tudo isso se mostra relevante no contexto desta pesquisa, pois o estado de saúde é um constructo social, e sua avaliação envolve uma compreensão cultural específica da pessoa (LAGUARDIA ET AL., 2011). 
Esta pesquisa foi apresentada ao Consulado-Geral do Brasil (na cidade do Porto, em Lisboa e em Faro), à Embaixada do Brasil em Lisboa e à Associação Mais Brasil (AMB). Esses órgãos apoiaram de modo significativo a identificação e abordagem de brasileiras que vivem em Portugal.

A coleta de dados ocorreu de julho a setembro de 2016, e foram instituídas duas modalidades: on-line, por meio da Plataforma LimeSurvey, e presencial, no Consulado-Geral do Brasil (na cidade do Porto e em Lisboa) e na AMB. Para complementar a abordagem on-line, foi criado um grupo no Facebook ${ }^{\circledR}$ : 'Brasileiras que Vivem em Portugal'. O link do inquérito do estudo foi disponibilizado nas páginas oficiais das redes sociais da AMB, do ConsuladoGeral do Brasil em Faro e da Embaixada do Brasil em Lisboa e no grupo no Facebook ${ }^{\circledR}$ 'Brasileiras que Vivem em Portugal'. A abordagem e identificação presencial foram desenvolvidas no Consulado-Geral do Brasil (na cidade do Porto e em Lisboa) e na AMB, com apresentação da pesquisa no que concerne aos objetivos, ao método adotado e à sua importância social. Em seguida, houve convite para a participação voluntária com vistas ao preenchimento do inquérito. Vale ressaltar que, em ambas as modalidades (online e presencial), a participação foi formalizada por meio da assinatura do Termo de Consentimento Livre e Esclarecido.

Os critérios de inclusão adotados foram: a) mulher brasileira; b) viver em Portugal há mais de três meses; e c) ter mais de 18 anos de idade. Vale destacar que este estudo foi desenvolvido no âmbito de uma pesquisa mais ampla, intitulada 'Estado de saúde e qualidade de vida de imigrantes brasileiras em Portugal', que obteve parecer favorável do Comitê de Ética em Pesquisa da Universidade Estadual Vale do Acaraú (UVA), sob o Protocolo n ${ }^{\circ} 1.692 .063$.

Os dados foram compilados na Plataforma LimeSurvey e processados no programa Statistical Package for the Social Sciences
(SPSS), versão 24.0. Recorreu-se à estatística descritiva com vistas a identificar o modo como as respostas são distribuídas (MARÔco, 2011), e a análise de consistência interna da escala teve por bases Cronbach (1951) e DeVellis (1991).

\section{Resultados}

A caracterização da amostra revelou, em relação à faixa etária, que 22,1\% tinham de 18 a 29 anos; 38,4\% tinham de 30 a 39 anos; 23,1\% tinham de 40 a 49 anos; e 15,5\% tinham de 50 a 76 anos. Quanto ao tempo de residência em Portugal, 20\% viviam no país há um ano; $25 \%$ entre dois e cinco anos; $29 \%$ entre seis e dez anos; e 26\% há mais de dez anos.

Tendo em vista o estado civil, $27 \%$ eram solteiras; $48 \%$ eram casadas; $13 \%$ viviam em união de fato; $2 \%$ eram separadas; $8 \%$ eram divorciadas; e $2 \%$ eram viúvas. A análise da nacionalidade dos cônjuges das brasileiras com marido ou companheiro em Portugal levantou os seguintes dados: $57 \%$ são portugueses; $37 \%$ são brasileiros; 2\% são luso-brasileiros; e 4\% são de outras nacionalidades, como franceses, angolanos, cabo-verdianos, espanhóis, alemães, britânicos, holandeses ou senegaleses.

Em relação à maternidade, 58,4\% tinham filho(s), e 41,6\% não tinham. Entre as mães, $45 \%$ tinham um filho; $40 \%$ tinham dois; $12 \%$ tinham três; $2 \%$ tinham quatro; e $1 \%$ tinha cinco filhos.

Ao considerar o nível de escolaridade, constatou-se que: $5 \%$ tinham ensino fundamental incompleto; $5 \%$ tinham ensino fundamental completo; $8 \%$ tinham ensino médio incompleto; $25 \%$ tinham ensino médio completo; $15 \%$ tinham ensino superior incompleto; $15 \%$ tinham ensino superior completo; e 27\% tinham pós-graduação.

Em termos de situação de moradia em Portugal, 69\% viviam com sua própria família; $15 \%$ viviam sozinhas; $6 \%$ viviam com amigos brasileiros; e 10\% viviam com uma família portuguesa. 
Por fim, a análise da amostra levantou o seguinte quadro no mercado de trabalho: $21 \%$ estavam desempregadas; $44 \%$ estavam empregadas; $17 \%$ eram estudantes; $5 \%$ eram tanto estudantes como trabalhadoras; e $13 \%$ eram domésticas ou donas de casa.

$\mathrm{Na}$ tabela 1, apresenta-se de forma detalhada cada item do SF-36 respondido pelas imigrantes brasileiras que vivem em Portugal e sua consistência. O valor do Alfa de Cronbach é superior ao valor de 0,80 , pelo que se pode considerar que as 36 variáveis medem de forma adequada uma única dimensão: a QV.

Tabela 1. Correlação item-total e efeito da eliminação de cada item do SF-36 aplicado a imigrantes brasileiras em Portugal

\begin{tabular}{|c|c|c|}
\hline & $\begin{array}{r}\text { Correlação } \\
\text { item-total } \\
\text { corrigida } \\
\end{array}$ & $\begin{array}{r}\text { Alfa de } \\
\text { Cronbach sem } \\
\text { o item }\end{array}$ \\
\hline $\begin{array}{l}\text { Atividades vigorosas, que exigem muito esforço, tais como correr, levantar obje- } \\
\text { tos pesados, participar de esportes árduos }\end{array}$ & 0,356 & 0,941 \\
\hline $\begin{array}{l}\text { Atividades moderadas, tais como mover uma mesa, passar aspirador de pó, jogar } \\
\text { bola, varrer casa }\end{array}$ & 0,471 & 0,940 \\
\hline Levantar ou carregar mantimentos & 0,500 & 0,940 \\
\hline Subir vários lances de escadas & 0,521 & 0,940 \\
\hline Subir um lance de escadas & 0,458 & 0,941 \\
\hline Curvar-se, ajoelhar-se ou dobrar-se & 0,524 & 0,940 \\
\hline Andar mais de $1 \mathrm{~km}$ & 0,491 & 0,940 \\
\hline Andar vários quarteirões & 0,433 & 0,940 \\
\hline Andar um quarteirão & 0,474 & 0,941 \\
\hline Tomar banho ou vestir-se & 0,257 & 0,941 \\
\hline $\begin{array}{l}\text { Você diminuiu a quantidade de tempo que dedicava ao seu trabalho ou a outras } \\
\text { atividades? }\end{array}$ & 0,450 & 0,941 \\
\hline Realizou menos tarefas do que você gostaria? & 0,479 & 0,940 \\
\hline Esteve limitada no seu tipo de trabalho ou a outras atividades? & 0,473 & 0,941 \\
\hline $\begin{array}{l}\text { Teve dificuldade de fazer seu trabalho ou outras atividades (por exemplo, neces- } \\
\text { sitou de um esforço extra)? }\end{array}$ & 0,573 & 0,940 \\
\hline Quanta dor no corpo você teve durante as últimas 4 semanas? & 0,653 & 0,938 \\
\hline $\begin{array}{l}\text { Durante as últimas } 4 \text { semanas, quanto a dor interferiu em seu trabalho normal } \\
\text { (incluindo o trabalho dentro de casa)? }\end{array}$ & 0,676 & 0,938 \\
\hline Em geral, o que você diria sobre sua saúde? & 0,496 & 0,940 \\
\hline Eu costumo adoecer um pouco mais facilmente que as outras pessoas & 0,521 & 0,940 \\
\hline Eu sou tão saudável quanto qualquer pessoa que conheço & 0,518 & 0,940 \\
\hline Eu acho que a minha saúde vai piorar & 0,557 & 0,939 \\
\hline Minha saúde é excelente & 0,659 & 0,938 \\
\hline $\begin{array}{l}\text { Por quanto tempo você tem se sentido cheia de vigor, cheia de vontade, cheia de } \\
\text { força? }\end{array}$ & 0,730 & 0,937 \\
\hline Por quanto tempo você tem se sentido com muita energia? & 0,707 & 0,938 \\
\hline Por quanto tempo você tem se sentido esgotada? & 0,704 & 0,938 \\
\hline Por quando tempo você tem se sentido cansada? & 0,714 & 0,938 \\
\hline
\end{tabular}


Tabela 1. (cont.)

\begin{tabular}{lc}
\hline $\begin{array}{l}\text { Durante as últimas } 4 \text { semanas, de que maneira sua saúde física ou problemas } \\
\text { emocionais interferiram em suas atividades sociais normais, em relação a família, } \\
\text { amigos ou grupo? }\end{array}$ & 0,729 \\
$\begin{array}{l}\text { Durante as últimas } 4 \text { semanas, por quanto tempo sua saúde física ou problemas } \\
\text { emocionais interferiram em suas atividades sociais (por exemplo, visitar amigos, } \\
\text { parentes etc.)? }\end{array}$ & 0,663 \\
$\begin{array}{l}\text { Você diminuiu a quantidade de tempo que dedicava ao seu trabalho ou a outras } \\
\text { atividades? }\end{array}$ & 0,501 \\
Realizou menos tarefas do que gostaria? & 0,525 \\
$\begin{array}{l}\text { Não realizou ou fez qualquer das atividades com tanto cuidado como geralmente } \\
\text { faz? }\end{array}$ & 0,529 \\
Por quanto tempo você tem se sentido uma pessoa muito nervosa? & 0,940 \\
Por quanto tempo você tem se sentido tão deprimida que nada pode animá-la? & 0,635 \\
Por quanto tempo você tem se sentido calma e tranquila? & 0,700 \\
Por quanto tempo você tem se sentido desanimada e abatida? & 0,939 \\
Por quanto tempo você tem se sentido uma pessoa feliz? & 0,938 \\
Em comparação a um ano atrás, como você classificaria sua saúde em geral? & 0,939 \\
\hline
\end{tabular}

A tabela 2 apresenta as nove dimensões agrupadas, e o valor do Alfa de Cronbach é superior ou está próximo do valor de 0,80 , pelo que se pode considerar que as variáveis incluídas em cada dimensão medem de forma adequada essa dimensão.

Tabela 2. Consistência interna por itens das dimensões do SF-36 aplicado a imigrantes brasileiras em Portugal

\begin{tabular}{lrr}
\hline & $\begin{array}{r}\text { Alfa de } \\
\text { Cronbach }\end{array}$ & $\begin{array}{r}\text { Número de } \\
\text { itens }\end{array}$ \\
\hline 1. Capacidade funcional & 0,903 & 10 \\
2. Aspectos físicos & 0,837 & 4 \\
3. Dor & 0,818 & 2 \\
4. Estado geral de saúde & 0,793 & 5 \\
5. Vitalidade & 0,868 & 4 \\
6. Aspectos sociais & 0,781 & 2 \\
7. Aspectos emocionais & 0,839 & 3 \\
8. Saúde mental & 0,872 & 5 \\
9. Mudança do estado de saúde ao longo do tempo & a & 1 \\
\hline
\end{tabular}

Nota: a - apenas tem um item. $O$ item b) da questão 11 apresenta correlação negativa com a subescala.

A tabela 3 converte os resultados mais baixos possíveis e os mais altos possíveis em 0 e 100 respectivamente. Os resultados que se situam entre esses valores representam o percentual total dos resultados possíveis alcançados. Quanto mais elevado o percentual obtido pelo sujeito, melhor será sua QV. Pode-se perceber que a vitalidade $60,6 \%$, saúde mental $66,3 \%$ e aspectos emocionais $68,8 \%$ são os níveis mais baixos das dimensões. 
Tabela 3. Médias dos domínios do SF-36 aplicado a imigrantes brasileiras em Portugal

\begin{tabular}{lcccccc}
\hline & N & Média & $\begin{array}{r}\text { Desvio } \\
\text { padrão }\end{array}$ & $\begin{array}{r}\text { Coeficiente de } \\
\text { variação }\end{array}$ & Mín & Máx \\
\hline SF-36 & 621 & 70,9 & 17,3 & $24 \%$ & 8 & 100 \\
1. Capacidade funcional & 670 & 83,6 & 18,8 & $22 \%$ & 0 & 100 \\
2. Aspectos físicos & 671 & 80,6 & 32,3 & $40 \%$ & 0 & 100 \\
3. Dor & 673 & 73,3 & 23,4 & $32 \%$ & 0 & 100 \\
4. Estado geral de saúde & 665 & 71,1 & 21,0 & $30 \%$ & 0 & 100 \\
5. Vitalidade & 676 & 60,6 & 22,8 & $38 \%$ & 0 & 100 \\
6. Aspectos sociais & 676 & 74,3 & 26,1 & $35 \%$ & 0 & 100 \\
7. Aspectos emocionais & 670 & 68,8 & 40,2 & $58 \%$ & 0 & 100 \\
8. Saúde mental & 667 & 66,3 & 21,7 & $33 \%$ & 0 & 100 \\
9. Mudança do estado de saúde ao longo & 679 & 56,1 & 22,7 & $40 \%$ & 0 & 100 \\
do tempo & & & & & & \\
\hline
\end{tabular}

Nota: Os valores indicados referem-se à escala de medida, entre 0\% e 100\%.

Verifica-se que a apreciação do estado de saúde, em média, é superior para a dimensão 1, 'Capacidade funcional' (83,6\%). Em seguida, têm-se as dimensões 2, 'Aspectos físicos' (80,6\%); 6, 'Aspectos sociais' (74,3\%); 3, 'Dor' (73,1\%); 4, 'Estado geral de saúde' (71,1\%); 7, 'Aspectos emocionais' (68,8\%); 8, 'Saúde mental' (66,3\%); 5, 'Vitalidade' (60,6\%); e, por fim, 9, 'Mudança de saúde ao longo do tempo' $(56,1 \%)$. Todas as dimensões apresentam valor médio superior ao ponto intermédio da escala.

No gráfico 1, pode-se observar que o SF-36 apresenta valor médio de 70,9\%, com dispersão de valores de $24 \%$. Os valores mínimo e máximo são, respectivamente, 0 e $100 \%$. Observa-se que a distribuição dos valores na escala do SF-36 está concentrada entre $50 \%$ e $80 \%$ e que todos os valores inferiores a $20 \%$ são considerados outliers, isto é, casos extremos que saem da distribuição normal.

Gráfico 1. SF-36 e seus respectivos domínios relacionados com a QV de imigrantes brasileiras em Portugal

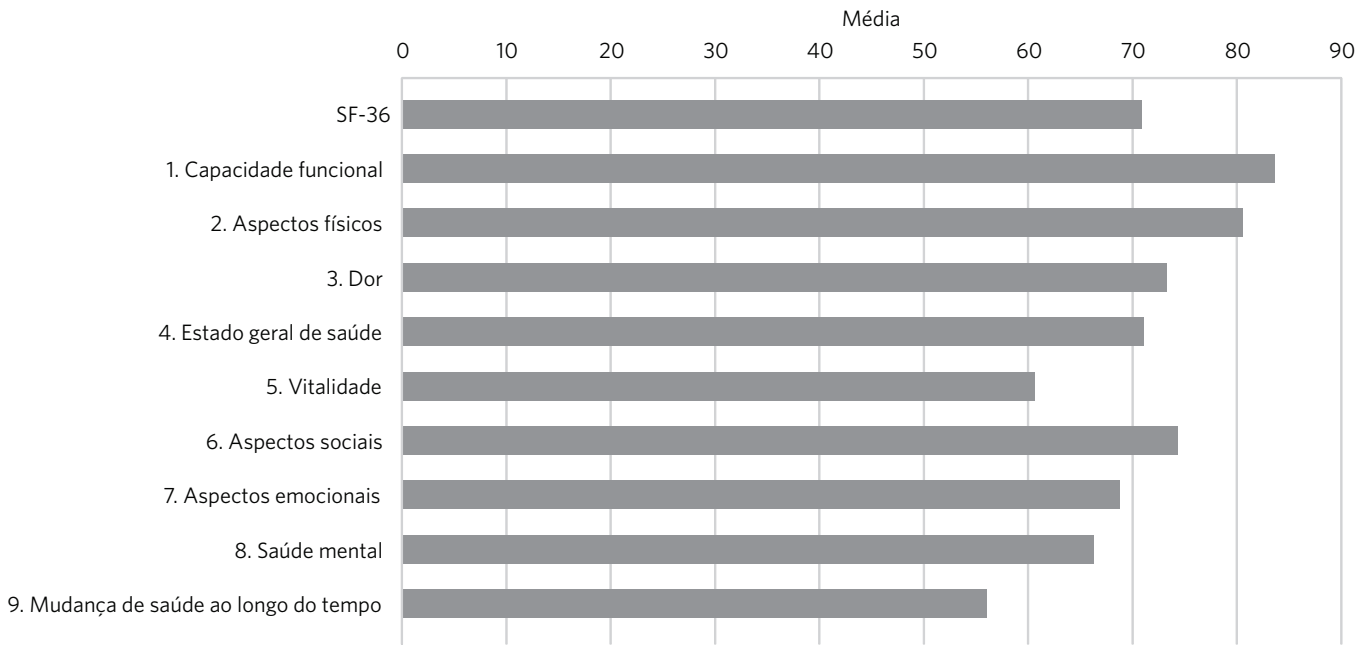




\section{Discussão}

Atualmente, as mulheres migram por motivos diversos: algumas fazem-no por razões econômicas, outras para assegurar a reunificação familiar ou escapar a constrangimentos culturais. No cenário atual de feminização da imigração, as mulheres imigram cada vez mais por sua conta e risco, em busca de emancipação e autonomia pessoal e econômica, não se resignando passivamente perante as múltiplas formas de discriminação de que são alvo, associando-se inclusivamente a outras mulheres com o intuito de chamar a si direitos fundamentais de exercício de cidadania (NEVES ET AL., 2016).

No que se refere à relação entre $\mathrm{a} Q \mathrm{QV}$ e a saúde, Ferreira, Diettrich e Pedro (2015) lembram que o ser humano busca indefinidamente a melhoria de suas condições de vida e o máximo de autonomia possível durante toda a sua existência. O processo migratório encontra-se em foco quando o assunto é QV e saúde, pois a maioria das pessoas que migram foi em busca de melhores condições de vida e saúde.

Pegoraro e Caldana (2008) ressaltam que a saúde física e mental feminina é afetada por seu contexto de vida ou por fatores externos, como aspectos socioculturais, legais, econômicos, de infraestrutura ou ambientais. Nesse cenário migratório de brasileiras em Portugal, os determinantes envolvidos no processo saúde doença parecem estar intimamente relacionados com a condição de imigrante.

Embora não tenha sido objeto de análise deste estudo, alguns aspectos da vida dessas mulheres brasileiras em Portugal devem ser levados em consideração, por estarem intimamente relacionados com sua condição de imigrante e, consequentemente, com os aspectos relativos com QV e saúde. Chama-se atenção para o estado civil e, mais especificamente, para a nacionalidade dos cônjuges; tempo de residência no país de acolhimento; número de filhos e destes quantos não estão vivendo com a mãe em Portugal; condições de inserção no mercado de trabalho e a situação de escolaridade. É inegável que todas essas variáveis influenciam de forma efetiva na QV de imigrantes. Recomendamse estudos que analisem a relação desses aspetos tendo como foco QV e saúde.

Em estudo sobre QV de imigrantes brasileiras em Portugal, os resultados revelaram que as que vivem sozinhas possuem níveis mais baixos de QV e saúde, em relação às que vivem acompanhadas. Morar sozinha parece ser uma condição, que vem crescendo, entre as mulheres que migram em busca de melhores condições de vida. Nesse mesmo estudo, os autores detectaram que as que estão desempregadas possuem mais baixos níveis de QV e saúde em relação às que estão em outra situação laboral ou inseridas de alguma forma no mercado de trabalho (OLIVEIRA ET AL., 2017).

A avaliação dos resultados obtidos por meio do SF-36, aplicado às imigrantes brasileiras que vivem em Portugal, proporciona a seguinte reflexão: as dimensões relacionadas com aspectos emocionais, saúde mental, vitalidade e mudança do estado de saúde ao longo do tempo apresentaram valores mais baixos, o que sugere a existência de condições que limitarão o desenvolvimento efetivo da QV nesse grupo no país de acolhimento.

A invisibilidade das mulheres na imigração foi o maior estímulo para a escolha desse tema de pesquisa, uma vez que poucos estudos enfocam essa população. Segundo Viana (2011), as mulheres devem ser levadas em consideração no desenvolvimento de estratégias da saúde e, também, no campo de investigação, seja devido à sua representatividade nos fluxos (mais da metade da comunidade brasileira), seja devido às questões específicas que são inerentes a elas (maior vulnerabilidade em relação à violência, exploração laboral e sexual).

Um estudo realizado com a comunidade brasileira em Lisboa identificou que o fato de apresentar ou não apresentar sofrimento psicológico esteve relacionado com gênero, longas jornadas de trabalho, doenças crônicas e QV. As mulheres se mostraram mais 
vulneráveis quanto aos aspectos emocionais, com índices mais baixos de saúde mental em comparação aos homens (VIANA, 2011).

Em Nante et al. (2016), as variáveis gênero, tempo de permanência e qualificação não estão associadas significativamente à QV sob o ponto de vista da saúde entre os imigrantes. Contudo, o efeito é significativo para as variáveis idade, país de procedência, idioma e cidade de acolhimento.

Reis e Ramos (2014), com utilização do World Health Organization Quality Of LifeBref (WHOQOL-Bref), constataram que homens e mulheres percebem e avaliam sua QV de modos diferentes. Em média, as mulheres avaliaram melhor sua $\mathrm{QV}$, ao passo que os homens avaliaram melhor a satisfação com sua saúde e os domínios físico, psicológico, das relações sociais e do meio ambiente.

Imigrantes do sexo feminino na Espanha, ao serem avaliadas com base no SF-36, apresentaram média superior ou igual a 60 em quase todas as dimensões, exceto nas funções físicas e emocionais e na vitalidade, em que os valores foram muito baixos. Esse mesmo grupo apresentou baixos níveis de QV nas dimensões física e emocional e na vitalidade, e foi identificado que se encontravam sob regime de trabalho interno sem contrato laboral e em situação irregular no país (BOVER ET AL., 2015).

Em pesquisa que analisou as diferenças de QV entre adolescentes imigrantes e nativos na Espanha e em Portugal, as mulheres relataram níveis mais baixos de $\mathrm{QV}$ do que os homens em termos de bem-estar físico (NUNES ET AL., 2016). Vale ressaltar a diferença entre os papéis tradicionais de gênero em determinadas sociedades, o que pode influenciar na QV e saúde de mulheres. Quanto mais desiguais as relações de gênero, aumenta a possibilidade de submissão, subordinação e todos os tipos de violências, gerando sentimentos negativos e comportamentos incapacitantes. Oliveira et al. (2017), em estudo com brasileiras em Portugal, identificou que as que estão morando há mais tempo no País possuem níveis mais adequados de QV e saúde.
Segundo Daher et al. (2011), as mulheres apresentaram níveis mais altos de problemas de saúde porque reagem de modo diferente a condições materiais, comportamentais e psicossociais de promoção da saúde. Elas tendem a ser menos empregadas em comparação aos homens e também vivenciam desigualdades em termos de renda e educação. A pontuação mais baixa entre as mulheres também pode ser explicada por variações hormonais, nascimento de filho(s) e o papel desempenhado como mãe e esposa, fatores observados com frequência nos relatos sobre as condições de saúde e a QV de imigrantes do sexo feminino.

De acordo com Pussetti (2010), geralmente a 'invisibilidade social' da experiência migratória é ampliada pelas contradições das políticas migratórias e pelas barreiras burocráticas, que geram perturbações emocionais e patologias mentais nessa população.

Um contraponto sobre a situação e condições de vida de imigrantes deve ser lembrado. De acordo com Neves et al. (2016), as mulheres migrantes enfrentam frequentemente situações de discriminação racial, étnica e religiosa, situando-se estas em um espectro que contempla desde formas sutis de exclusão até manifestações públicas de racismo e xenofobia. Se por um lado as mulheres imigrantes procuram ativamente consagrar as conquistas obtidas em termos de direitos, ocupando os diferentes espaços da vida social em igualdade de circunstâncias, por outro, continuam a ver-se confrontadas com barreiras conjunturais que as limitam nas suas escolhas e oportunidades.

Ao identificar que os aspectos emocionais, saúde mental, vitalidade e mudança de saúde ao longo do tempo são as dimensões que estão mais vulneráveis no contexto da QV dessas imigrantes, pode-se supor que a forma como são recebidas e tratadas no país de acolhimento está refletindo diretamente em seus sentimentos e comportamentos.

Alguns estudos realizados, como os de Franken, Coutinho e Ramos (2012), demonstraram a presença de sofrimento psíquico e 
adoecimento mental na população de imigrantes, e as mulheres em geral são acometidas em maior proporção do que os homens.

Nesse cenário que envolve a QV e saúde de mulheres brasileiras em Portugal, os aspectos relacionados com a saúde física e mental da população estudada são componentes importantes que merecem intervenção e monitoramento sistemático.

\section{Conclusões}

A imigração, por si, não pode ser identificada como determinante de doença, sofrimento psicológico e diminuição da QV. É certo que migrar coloca desafios para o ser humano, ao passo que também pode gerar oportunidades. Nessa perspectiva, este artigo apresenta um diagnóstico preliminar de situações enfrentadas por brasileiras que decidiram viver em Portugal.

As dimensões do SF-36, no que concerne a capacidade funcional, aspectos físicos, dor, estado geral de saúde, vitalidade, aspectos sociais, aspectos emocionais, saúde mental e mudança do estado de saúde ao longo do tempo, proporcionam conhecimentos valiosos para a compreensão das formas de vida e sobrevivência no país de acolhimento.

O estudo evidencia as dimensões com os mais baixos percentuais, identificadas como as mais vulneráveis no contexto da QV de imigrantes, estas são: aspectos emocionais $68,8 \%$, saúde mental $66,3 \%$; vitalidade 60,6\%; mudança de saúde ao longo do tempo $56,1 \%$. Esses resultados são importantes para investimentos em políticas públicas e para outros estudos com foco nessas dimensões. A saúde física e mental deve ser objeto de intervenção e cuidado, pois o estudo revela que a condição de imigrante brasileira em Portugal enfraqueceu algumas dimensões de sua QV e saúde.

Pode-se apontar como limitações deste estudo o fato de não recorrer a um aprofundamento qualitativo, no qual o contexto e as condições de vida poderiam ser apresentados de modo subjetivo, enriquecendo o diagnóstico apresentado. Esta investigação oferece insights sobre os domínios para a mensuração da QV de imigrantes brasileiras em Portugal, já que foram detectados valores menores nas dimensões relacionadas com aspectos emocionais, saúde mental, vitalidade e mudança do estado de saúde ao longo do tempo, apesar de haver necessidade de aprofundamento quanto ao contexto social e às formas de vida.

Sugerem-se estudos qualitativos destinados a informar exatamente quais seriam as circunstâncias que efetivamente incidem na diminuição da QV, no que diz respeito aos aspectos emocionais, saúde mental, vitalidade e mudança do estado de saúde ao longo do tempo. Isso porque cada um desses parâmetros congrega uma quantidade ampla de fatores, o que dificultaria a identificação de pontos em que as políticas públicas de imigração e os processos de acolhimento possam ser aprimorados.

Acredita-se ser fundamental a criação de uma política que tenha por objetivo ações transformadoras voltadas ao fortalecimento da consciência cidadã e coletiva dessas imigrantes brasileiras, gerando melhores condições para o exercício pleno da cidadania no país de acolhimento.

Por fim, sintetiza-se este artigo por meio de uma mensagem de Malheiros (2007, P. 30):

esperem encontrar, neste trabalho, verdades temporariamente verificáveis, ficando a cargo das investigações posteriores completar, aprofundar ou mesmo refutar o que aqui foi escrito.

\section{Colaboradores}

Eliany Nazaré Oliveira participou substancialmente da concepção, planejamento, análise e interpretação dos dados e escrita da versão final do manuscrito. 
Félix Fernando Monteiro Neto contribui de forma efetiva para a concepção, planejamento, análise, interpretação dos dados e escrita do manuscrito.

Tamires Alexandre Félix contribuiu para a revisão crítica do conteúdo e participou da revisão da versão final do manuscrito.

\section{Referências}

BÄCKSTRÖM, B.; PEREIRA, S. C. A questão migratória e as estratégias de convivência entre culturas diferentes em Portugal. Revista Interdisciplinar da Mobilidade Humana, Brasília, DF, v. 20, n. 38, p. 83-100, 2012.

BOVER, A. et al. Calidad de vida de trabajadoras inmigrantes latinoamericanas como cuidadoras en España. Gaceta Sanitaria, Barcelona, v. 29, n. 2, p. 123-126, 2015.

CICONELLI, R. M. Tradução para o português e validação do questionário genérico de avaliação de qualidade de vida. 1997. 120 f. Tese (Doutorado em Ciências Médicas) - Escola Paulista de Medicina, Universidade Federal de São Paulo, São Paulo, 1997.

CORREA, M. A. S. et al. Migração por sobrevivência: soluções brasileiras. Revista Interdisciplinar da Mobilidade Humana, Brasília, DF, v. 23, n. 44, p. 221236, 2015.

CRONBACH, L. J. Coefficient alpha and the internal structure of tests. Psychometrika, [S. 1.], v. 16, p. 297-334, 1951.

DAHER, A. M. et al. Health related quality of life among Iraqi immigrants settled in Malaysia. BMC Public Health, Londres, v. 11, p. 407, 2011.
Gleisson Ferreira Lima participou da coleta de dados e contribuiu para a revisão final do conteúdo do manuscrito.

Paulo César de Almeida realizou o tratamento estatístico dos dados e contribuiu para a revisão final do conteúdo do manuscrito
DEVELLIS, R. F. Scale development.: theory and applications. London: Sage, 1991.

FERREIRA, J. S.; DIETTRICH, S. H. C.; PEDRO, D. A. Influência da prática de atividade física sobre a qualidade de vida de usuários do SUS. Saúde em Debate, Rio de Janeiro, v. 39, n. 106, p. 792-801, jul./set. 2015.

\section{FRANKEN, I.; COUTINHO, M. P. L.; RAMOS, M. N.} P. Representações sociais, saúde mental e imigração internacional. Psicologia: Ciência Profissão, Brasília, DF, v. 32, n. 1, p. 202-219, 2012.

LAGUARDIA, J. et al. Psychometric evaluation of the SF-36 (v.2) questionnaire in a probability sample of Brazilian households: results of the survey. Pesquisa Dimensões Sociais das Desigualdades (PDSD), Brazil 2008. Health and Quality of Life Outcomes, Londres, v. 9, p. 61, ago. 2011.

MALHEIROS, J. Os brasileiros em Portugal: a síntese do que sabemos. In: MALHEIROS, J. (Ed.). A imigração brasileira em Portugal. Lisboa: Alto Comissariado para a Imigração e Diálogo Intercultural, 2007. p. 11-37.

MARINUCCI, R. Migrações internacionais e processos comunicacionais. Revista Interdisciplinar da Mobilidade Humana, Brasília, DF, v. 24, n. 46, p. 7-10, jan./abr. 2016. 
MARÔCO, J. Análise estatística com o SPSS Statistics. 5. ed. Pero Pinheiro: Report Number, 2011.

MEZZADRA, S. Multiplicação das fronteiras e das práticas de mobilidade. Revista Interdisciplinar da Mobilidade Humana, Brasília, DF, v. 23, n. 44, p. 11-30, jan./jun. 2015.

MINAYO, M.; HARTZ, Z. M. A.; BUSS, P. M. Qualidade de vida e saúde: um debate necessário. Ciência \&t Saúde Coletiva, Rio de Janeiro, v. 5, n. 1, p. 7-18, 2000.

NAIME, J. O perfil da migração africana. Conjuntura Internacional, Belo Horizonte, v. 3, p. 11-16, 2006.

NANTE, N. L. G. et al. Quality of life in refugees and asylum seekers in Italy: a pilot study. Annali dell'Istituto Superiore di Sanità, Roma, v. 52, n. 3, p. 424-427, 2016.

NEVES, A. S. A. et al. Mulheres imigrantes em Portugal: uma análise de gênero. Estudos de Psicologia, Campinas, v. 33, n. 4, p. 723-733, out./dez. 2016.

NUNES, C. et al. Quality of life of Portuguese and Spanish adolescents. A comparative study between natives and immigrants. Ciência \&t Saúde Coletiva, Rio de Janeiro, v. 21, n. 4, p. 1137-1144, 2016.

OLIVEIRA, E. N. et al. Migration, Quality of life and health of brazilian immigrants in Portugal. International Archives of Medicine, Bethesda, v. 10, n. 158, p. 1-13, maio 2017.

PEGORARO, R. F.; CALDANA, R. H. L. Mulheres, loucura e cuidado: a condição da mulher na provisão e demanda por cuidados em saúde mental. Saúde e Sociedade, São Paulo, v. 17, n. 2, p. 82-94, abr./jun. 2008.
PEROTTI, A. Apologia do intercultural. Lisboa:

Secretariado Coordenador dos Programas de Educação Multicultural, 1997.

PUSSETTI, C. Identidades em crise: imigrantes, emoções e saúde mental em Portugal. Saúde e Sociedade, São Paulo, v. 19, n. 1, p. 94-113, jan./mar. 2010.

RAMOS, N. Maternage en milieu portugais autochtone et immigré, de la tradition à la modernité: une étude ethnopsychologique. 1993. 736 f. Tese. (Doutorado em Psicologia) - Universidade de Paris V, Paris, 1993.

Adaptação, saúde e doença em contexto migratório e intercultural. In: RAMOS, N. (Org.). Psicologia clínica e da saúde. Lisboa: Universidade Aberta, 2004. p. 239-299.

REIS, L.; RAMOS, N. Adaptação, saúde e qualidade de vida de imigrantes brasileiros residentes na região de Lisboa. In: HITA-YÁÑEZ, E.; DÍAZ-ROMÁN, A.; RAMIRO, M. T. (Org.). Avances en psicología de la salud. Granada: Asociación Española de Psicología Conductual, 2014. p. 35-43.

VIANA, V. R. C. Saúde mental, qualidade de vida e acesso aos cuidados de saúde na comunidade brasileira de Lisboa. Lisboa: Alto Comissariado para a Imigração e Diálogo Intercultural, 2011.

WORLD HEALTH ORGANIZATION (WHO).

Enfermities full attention manual prevailing of childhood (AIEPI) and for studeats: preliminal version. Genebra: WHO, 2002

Recebido para publicação em janeiro de 2017

Versão final em junho de 2017

Conflito de interesses: inexistente

Suporte financeiro: não houve 